\title{
Anxiety in Parkinson's disease is associated with changes in the brain fear circuit
}

Citation for published version (APA):

Carey, G., Lopes, R., Viard, R., Betrouni, N., Kuchcinski, G., Devignes, Q., Defebvre, L., Leentjens, A. F. G., \& Dujardin, K. (2020). Anxiety in Parkinson's disease is associated with changes in the brain fear circuit. Parkinsonism \& Related Disorders, 80, 89-97. https://doi.org/10.1016/j.parkreldis.2020.09.020

Document status and date:

Published: 01/11/2020

DOI:

10.1016/j.parkreldis.2020.09.020

Document Version:

Publisher's PDF, also known as Version of record

Document license:

Taverne

Please check the document version of this publication:

- A submitted manuscript is the version of the article upon submission and before peer-review. There can be important differences between the submitted version and the official published version of record.

People interested in the research are advised to contact the author for the final version of the publication, or visit the DOI to the publisher's website.

- The final author version and the galley proof are versions of the publication after peer review.

- The final published version features the final layout of the paper including the volume, issue and page numbers.

Link to publication

\footnotetext{
General rights rights.

- You may freely distribute the URL identifying the publication in the public portal. please follow below link for the End User Agreement:

www.umlib.nl/taverne-license

Take down policy

If you believe that this document breaches copyright please contact us at:

repository@maastrichtuniversity.nl

providing details and we will investigate your claim.
}

Copyright and moral rights for the publications made accessible in the public portal are retained by the authors and/or other copyright owners and it is a condition of accessing publications that users recognise and abide by the legal requirements associated with these

- Users may download and print one copy of any publication from the public portal for the purpose of private study or research.

- You may not further distribute the material or use it for any profit-making activity or commercial gain

If the publication is distributed under the terms of Article $25 \mathrm{fa}$ of the Dutch Copyright Act, indicated by the "Taverne" license above, 


\title{
Anxiety in Parkinson's disease is associated with changes in the brain fear circuit
}

\author{
Guillaume Carey $^{\mathrm{a}, *}$, Renaud Lopes ${ }^{\mathrm{a}, \mathrm{c}}$, Romain Viard ${ }^{\mathrm{a}, \mathrm{c}}$, Nacim Betrouni ${ }^{\mathrm{a}}$, \\ Gregory Kuchcinski $^{\text {a,c }}$, Quentin Devignes ${ }^{a}$, Luc Defebvre ${ }^{a, b}$, Albert F.G. Leentjens ${ }^{\text {d }}$, \\ Kathy Dujardin ${ }^{\mathrm{a}, \mathrm{b}}$ \\ ${ }^{a}$ Univ. Lille, Inserm, CHU Lille, Lille Neurosciences and Cognition, Lille, France \\ ${ }^{\mathrm{b}}$ Neurology and Movement Disorders Department, Lille University Medical Centre, Lille, France \\ ${ }^{\mathrm{c}}$ Department of Neuroradiology, Lille University Medical Centre, Lille, France \\ ${ }^{\mathrm{d}}$ Department of Psychiatry, Maastricht University Medical Centre, Maastricht, the Netherlands
}

\section{A R T I C L E I N F O}

\section{Keywords:}

Parkinson's disease

Anxiety disorders

Fear circuit

Neuroimaging

Amygdala

\begin{abstract}
A B S T R A C T
Background: Anxiety is frequent in Parkinson's disease (PD) and has a negative impact on disease symptoms and quality of life. The underlying mechanisms remain largely unknown. The aim of this study was to identify anatomical and functional changes associated to PD-related anxiety by comparing the volume, shape and texture of the amygdala, the cortical thickness as well as the functional connectivity (FC) of the fear circuit in patients with and without clinically relevant anxiety.

Methods: Non-demented PD patients were recruited, and anxiety was quantified using the Parkinson Anxiety Scale. Structural MRI was used to compare cortical thickness and amygdala structure and resting-state functional MRI to compare FC patterns of the amygdala and resting-state functional networks in both groups.

Results: We included 118 patients: 34 with $(\mathrm{A}+$ ) and 84 without (A-) clinically relevant anxiety. Clusters of cortical thinning were identified in the bilateral fronto-cingulate and left parietal cortices of the $\mathrm{A}+$ group. The texture and the shape of the left amygdala was different in the A+ group but the overall volume did not differ between groups. FC between the amygdala and the whole brain regions did not differ between groups. The internetwork resting-state FC was higher between the "fear circuit" and salience network in the A+ group. Conclusion: Anxiety in PD induces structural modifications of the left amygdala, atrophy of the bilateral frontocingulate and the left parietal cortices, and a higher internetwork resting-state FC between the fear circuit and the salience network.
\end{abstract}

\section{Introduction}

Anxiety is among the most frequent non-motor symptoms in Parkinson's disease (PD) with an average point prevalence of $31 \%$ [1]. However, the underlying mechanisms remain poorly understood. In PD patients, studies have shown a negative correlation between the level of anxiety and the volume of the left amygdala, the anterior cingulate cortex (ACC) and precuneus thickness [2,3]. Resting-state functional MRI (rs-fMRI) studies have shown that the severity of anxiety was correlated with increased functional connectivity (FC) between the amygdala and the prefrontal cortex (PFC), as well as the temporal and parietal cortices, and the striatum [4,5]. These studies mostly correlated imaging data with anxiety levels, which probably reflects trait anxiety (i. e. the individual's tendency to experience anxiety) [6], but they did not compare patients with and without anxiety at the time of assessment, in order to reveal the mechanisms of state anxiety. Nevertheless, these studies suggest dysfunction of the fear circuit.

Based on animal studies, the existence of an anatomo-functional network called the "fear circuit" was postulated [7] whose hub is the amygdala [8]. In humans, the amygdala is known to be an interface between external stimuli and behavioral as well as cognitive responses to anxiety. Functional connections between the amygdala and the anterior cingulate cortex (ACC), the medial prefrontal cortex (mPFC), the insular cortex (IC), the hippocampus and the striatum have been reported, that together form the human "fear circuit" [9].

The aim of this study was to identify anatomical and functional changes associated with PD-related anxiety by comparing the volume,

\footnotetext{
* Corresponding author. CHU de Lille, Hôpital Roger Salengro, Service de Neurologie A, Avenue du Professeur Emile Laine, 59037, Lille, France.

E-mail addresses: guillaume.carey@chru-lille.fr, guillaume.carey.etu@univ-lille.fr (G. Carey).
} 


\begin{tabular}{|c|c|c|c|}
\hline \multicolumn{2}{|c|}{ Abbreviations } & LARS & Lille Apathy Rating Scale \\
\hline $3 \mathrm{D}-\mathrm{T}_{1} \mathrm{w}$ & 3-Dimension $\mathrm{T}_{1}$-weighted & MDRS & Mattis dementia rating scale \\
\hline & patient group with anxiety & MDS-UP & DRS Movement Disorder Society Unified Parkinson Disease \\
\hline A- & patient group without anxiety & & Rating Scale \\
\hline ACC & Anterior Cingulate Cortex & $\mathrm{mPFC}$ & medial prefrontal cortex \\
\hline BJLO & Benton judgment of line orientation test & PAS & Parkinson Anxiety Scale \\
\hline BNT & Boston naming test & PFC & prefrontal cortex \\
\hline dlPFC & dorsolateral prefrontal cortex & ROI & region of interest \\
\hline FDR & false discovery rate & rs-fMRI & resting state functional MRI \\
\hline GLCM & Grey-Level Co-occurrence Matrix & SDMT & Symbol digit modalities test \\
\hline HAMD & Hamilton Depression Rating Scale & TMT & Trail Making Test \\
\hline HVLT & Hopkins verbal learning test & vlPFC & ventrolateral prefrontal cortex \\
\hline IC & insular cortex & WAIS-R & Wechsler Adult Intelligence Scale - Revised. \\
\hline ICA & Independent Component Analyses & & \\
\hline
\end{tabular}

shape and texture of the amygdala, the cortical thickness, the FC of amygdala and the internetwork resting state FC of the fear circuit in PD patients with and without clinically relevant anxiety. We assumed that changes in the fear circuit will be observed in patients with anxiety, more specifically we hypothesized a smaller volume of the amygdala, a smaller cortical thickness of the prefrontal, cingulate and insular cortices and a higher FC within the fear circuit, compared to patients without anxiety.

\section{Materials and methods}

\subsection{Population}

This study included 156 consecutive PD patients enrolled from two movement disorders clinics in Lille (France) and Maastricht (The Netherlands) between March 2013 and August 2014. PD was diagnosed according to the United Kingdom Parkinson's Disease Society Brain Bank diagnostic criteria. Patients with other neurological disorders, as well as patients with moderate to severe dementia according to the Movement Disorders Society criteria for Parkinson's disease dementia were excluded [10].

Age, sex, duration of formal education, disease duration, history of PD or psychiatric disorders were recorded. The Movement Disorder Society Unified Parkinson Disease Rating Scale (MDS-UPDRS) was used to assess motor (part III), non-motor symptoms (part I) and disease severity (Hoehn-Yahr stage). The levodopa equivalent daily dosages (LEDD) were calculated and the use of antidepressant and anxiolytics treatments reported. Anxiety, depression and apathy were respectively assessed by the Parkinson Anxiety Scale (PAS) [11], the Hamilton Depression Rating Scale (HAMD) and the Lille Apathy Rating Scale (LARS). Each patient had undergone a thorough evaluation of cognitive functions (see Dujardin et al. [12] for details of the procedure). This evaluation assessed the overall cognitive efficiency (Mattis dementia rating scale (MDRS)); attention and working memory (symbol digit modalities test (SDMT), forward-backward digit span subtest of the Wechsler for adults intelligence scale revised (WAIS-R)); executive functions (Trail Making Test (TMT), phonemic and alternating fluency tests); episodic memory (Hopkins verbal learning test (HVLT)); language (Boston naming test (BNT) and animal fluency) and visuospatial functions (Benton judgment of line orientation test (BJLO)).

Written informed consent was obtained from all participants after full information of the procedure. The study was approved by the institutional ethics committees (CPP Nord-Ouest IV, 2012-A 01317-36). Additional information on this study group is detailed in the original paper [12].

\subsection{Characterization of anxiety}

Patients were divided into two groups, one with $(\mathrm{A}+)$ and one without (A-) anxiety, according to their score on the PAS, a scale specifically developed to detect anxiety in PD patients. We used the observer-rated version. Patients were considered "A+" if they had a score above the cut-off in at least one of the three subparts of the scale (part A (persistent anxiety) $>9$, part B (episodic anxiety) $>3$, or part C (avoidance behavior) $>3$ ) [11].

\subsection{Imaging data acquisition}

Patients were scanned at two sites using identical 3T Philips Achieva MRI scanner (Philips Healthcare, Best, The Netherlands) with identical software versions and MR sequences. High-resolution $3 \mathrm{D} \mathrm{T}_{1}$-weighted $\left(3 \mathrm{D}-\mathrm{T}_{1} \mathrm{w}\right)$ images were acquired with a magnetization-prepared gradient echo sequence (voxel size: $1 \mathrm{x} 1 \times 1 \mathrm{~mm}^{3}$; TR: $7.2 \mathrm{~ms}$; TE: $3.3 \mathrm{~ms}$; matrix size: 172 x 256 x 256 voxels; flip angle: $9^{\circ}$ ). rs-fMRI was performed with a 10 min $\mathrm{T}_{2}{ }^{*}$-weighted EPI sequence (voxel size: $3 \times 3 \times 3 \mathrm{~mm}^{3}$; TR: $2400 \mathrm{~ms}$; TE: $30 \mathrm{~ms}$; matrix size: $64 \times 64$ x 40 voxels; flip angle: $90^{\circ}$ ). Resting-state fMRI using posterior to anterior direction with interleaved acquisition was used. Patients were required to remain quiet, stay awake and close their eyes.

\subsection{Structural MRI analysis}

\subsubsection{Cortical thickness extraction}

To study potential cortical atrophy, cortical thickness was automatically calculated using FreeSurfer 5.3 software (https://surfer.nmr.mgh. harvard.edu/) from fMRIPrep tool [13]. Statistical analysis was implemented in Surf-Stat toolbox (http://www.math.mcgill.ca/keith/surfst at/) for MATLAB. The pipeline is detailed on Supplementary material, 1.a.

\subsubsection{Volume, shape and texture of amygdala}

Amygdala were manually segmented on $3 \mathrm{D}-\mathrm{T}_{1} \mathrm{~W}$ images with MRICRON software to bring out any atrophy. Anatomical borders were defined by a radiologist and according to literature data [14]. Volumes were recorded in $\mathrm{mm}^{3}$ and normalized to the patient's total intracranial volume estimated by FreeSurfer 5.3 software. The detailed procedure is provided in Supplementary material, 1.b.

To study potential deformations of the amygdala, shape analysis was performed using the spherical harmonic-point discrimination model (SPHARM-PDM) [15].

A texture analysis was performed on the $3 \mathrm{D}-\mathrm{T}_{1} \mathrm{w}$ images in order to determine changes in the amygdala. Texture analysis is an image processing method for the quantification of grey levels inside an image 
[16]. The procedure is detailed in Betrouni et al. [17]. Here, we compared four first-order and $7 \mathrm{~s}$-order texture parameters detailed in Supplementary Table 1.

\subsection{Functional MRI analysis}

\subsubsection{Preprocessing and quality control}

Common preprocessing steps, including co-registration, normalization, unwarping, noise component extraction, segmentation, skull stripping, slice-timing correction, were performed using fMRIprep 1.2.5 (https://fmriprep.readthedocs.io). At the end of this procedure, an individual quality control was performed. CONN Toolbox [18] was then used for: i) Gaussian kernel $6 \mathrm{~mm}$ smoothing; ii) to remove motion, physiological and other artefactual effects from BOLD signal; iii) Band-pass filter of $0.008 \mathrm{~Hz}-0.09 \mathrm{~Hz}$ (more details in Supplementary material 1.d).

\subsubsection{Resting-state functional connectivity of the amygdala}

Resting-state FC analysis were performed with CONN. A complete brain parcellation including 91 cortical areas and 15 subcortical areas from the FSL Harvard-Oxford Atlas [19] was used to define both amygdala and the regions of interest (ROI) in MNI-space. The right and left amygdala were defined as seeds and compared to all the other ROIs. The correlation indices between the mean BOLD signal of both amygdala and of the ROIs were calculated for each patient. These correlation indices were then compared in each group and between groups.

\subsubsection{Independent component analysis and functional network} connectivity

Group Independent Component Analyses (ICA) were performed to identify common functional networks in patients using Calhoun's grouplevel ICA approach with CONN. Forty independent components have been identified. A functional networks atlas from the Human Connectome Project, provided by the CONN Toolbox, was used to correlate common healthy functional networks with these forty components. The following networks were identified: default-mode network, left and right frontoparietal network (cognitive control), visual network, sensorimotor network and dorsal attentional network. Moreover, the salience network provided by default from the CONN toolbox was used because the group ICA failed to identify it. Finally, the "fear circuit" was defined using bilateral cortical and subcortical areas from the FSL Harvard-Oxford Atlas including amygdala, striatum, hippocampus, ACC, mPFC and IC [9]. Network masks were extracted. The correlation between the mean BOLD signal of these masks were calculated for each patient and compared in each group and between groups.

\subsection{Statistical analyses}

For all analyses, the statistical significance threshold was set at pvalue $<0.05$. Correction for multiple comparisons (FDR - False Discovery rate) were performed separately for cognitive variables, functional and structural data. The normality of distribution was assessed using a Kolmogorov-Smirnov test.

\subsubsection{Analysis of clinical data}

The numerical variables were described as means and standard deviations, the ordinal variables as median and range and the categorical variables as frequencies and percentages.

Qualitative data were compared using Odds Ratio's and quantitative data using two sample T-tests or Mann-Whitney tests depending on normality of the distribution. These analyses were performed with the Statistical Package for the Social Sciences, version 22 (SPSS, Chicago).

\subsubsection{MRI analyses}

Generalized linear models (GLM) were performed to compare cortical thickness, amygdala volumes and amygdala texture parameters between groups. Amygdala shape comparison was performed using a MANCOVA procedure. For rs-fMRI analyses, generalized linear models with permutation inference were calculated to identify significant functional connections for each group and to compare these connections between the groups.

\subsubsection{Regression analysis}

Hierarchical multiple regression post-hoc analyses were performed to examine the relationship between the PAS score and sub-scores and the volume and texture of amygdala, mean cortical thickness in the significant clusters and FC values. Center, sex and Hoehn-Yahr stage were set as nuisance regressors in the first block (model 1) of all regression models whereas PAS score or sub-scores (independent variable) were separately added to the second block (model 2) of the model to examine the association between anxiety symptoms and imaging data, adjusted by the effect of center, sex and Hoehn-Yahr stage. We ensured that all models met the assumptions of multiple regression analyses, including normality of the residuals, multicollinearity and homoscedasticity.

\section{Results}

\subsection{Population}

After exclusion of 38 patients for dementia $(n=14)$, refusal or contraindication to have an MRI $(\mathrm{n}=22)$ or unusable MRI (major motion artefact $-\mathrm{n}=2$ ), 118 were involved in the present study, 34 with ("A+") and 84 without ("A-") anxiety (Flowchart in Supplementary Fig. 1).

\subsection{Demographic and clinical variables}

"A+" patients were more frequently female, with a family history of $\mathrm{PD}$, and more often a left-sided onset of motor symptoms. MDS-UPDRS part I sub-scores of depression, anxiety, sleep disturbances, pain, and fatigue were higher in " $\mathrm{A}+$ " group than in the "A-" group and disease stage was more advanced. In the "A+" group, LEDD was higher and antidepressants and anxiolytics were used more frequently (Table 1).

\subsection{Cognitive variables}

After FDR-correction for multiple comparisons, patients in the "A+" group had lower results at backward digit span and animal fluency test as well as a slower processing speed than in the "A-" group (Table 1).

\subsection{Structural analyses}

All following analyses were adjusted for sex, disease stage and center.

\subsubsection{Cortical thickness}

Three clusters of reduced cortical thickness were identified in the bilateral frontal and left parietal regions in the "A+" compared to "A-" group (Fig. 1). There was no significant difference for the reverse analysis.

\subsubsection{Volume, shape and texture of amygdala}

There was no significant group difference for the volume of amygdala (A+/A-, left: $1201 \mathrm{~mm}^{3} / 1284 \mathrm{~mm}^{3}$; right: $1210 \mathrm{~mm}^{3} / 1273 \mathrm{~mm}^{3}$ ). Shape analysis revealed several remodeling areas located on the medial and postero-lateral sides of the left amygdala. Texture analyses showed a significant group difference for the second-order texture value "correlation", in the left amygdala ( $\mathrm{F}=3.86, \mathrm{p}=0.025$ ) (Fig. 2). 
Table 1

Demographic, clinical and cognitive variables: group comparisons (Parkinson's disease patients with (A+) and without (A-) anxiety).

\begin{tabular}{|c|c|c|c|c|}
\hline Demographic variables & $\mathrm{A}+\operatorname{group}(\mathrm{n}=34)$ & A- group $(n=84)$ & \multicolumn{2}{|l|}{ OR (CI 95\%); p-value } \\
\hline Age (years) & $65.62( \pm 7.66)$ & $64.10( \pm 8.62)$ & \multicolumn{2}{|l|}{0.37} \\
\hline Women $(\mathrm{n}=36)$ & $16(47.06 \%)$ & $20(23.81 \%)$ & \multicolumn{2}{|c|}{$2.84(1.23 ; 6.58) ; p=0.013^{a}$} \\
\hline Hand dominance (right, $\mathrm{n}=101$ ) & $29(85.29 \%)$ & $72(85.71 \%)$ & \multicolumn{2}{|c|}{$0.97(0.31 ; 3) ; \mathrm{p}=0.99$} \\
\hline Formal education (years) & $12.12( \pm 3.96)$ & $12.61( \pm 3.53)$ & \multicolumn{2}{|c|}{0.51} \\
\hline Illness duration (years) & $9.59( \pm 7.82)$ & $8.18( \pm 4.99)$ & \multicolumn{2}{|l|}{0.25} \\
\hline \multicolumn{3}{|l|}{ Left $(n=48)$} & \multicolumn{2}{|c|}{$3.21(1.31 ; 7.85) ; p=0.009^{a}$} \\
\hline \multicolumn{5}{|l|}{ Clinical variables } \\
\hline LEDD (mg/day) & $937.36( \pm 494.38)$ & $732.53( \pm 578.12)$ & \multicolumn{2}{|l|}{$0.02^{\mathrm{a}}$} \\
\hline Antidepressant use $(\mathrm{n}=17)$ & $13(38.24 \%)$ & $4(4.76 \%)$ & \multicolumn{2}{|c|}{$12.38(3.66 ; 41.91) ; \mathrm{p}<0.0001^{\mathrm{a}}$} \\
\hline Anxiolytic use $(n=12)$ & $10(29.41 \%)$ & $2(2.38 \%)$ & \multicolumn{2}{|c|}{$17.08(3.5 ; 83.33) ; \mathrm{p}<0.0001^{\mathrm{a}}$} \\
\hline \multicolumn{5}{|l|}{ MDS-UPDRS part $1(0-4) s$} \\
\hline 1.3. Depressed mood & $1(0-4)$ & $0(0-4)$ & \multicolumn{2}{|l|}{$0.001^{\mathrm{a}}$} \\
\hline 1.4. Anxious mood & $2(0-4)$ & $0(0-4)$ & \multicolumn{2}{|l|}{$<0.0001^{\mathrm{a}}$} \\
\hline 1.7. Night-time sleep problems & $2(0-4)$ & $2(0-4)$ & \multicolumn{2}{|l|}{$0.03^{\mathrm{a}}$} \\
\hline 1.8. Daytime sleepiness & $2(0-4)$ & $2(0-4)$ & \multicolumn{2}{|l|}{0.26} \\
\hline 1.9. Pain and other sensations & $2(0-4)$ & $1(0-4)$ & \multicolumn{2}{|l|}{$<0.0001^{\mathrm{a}}$} \\
\hline 1.10. Urinary problems & $1(0-4)$ & $1(0-4)$ & \multicolumn{2}{|l|}{0.09} \\
\hline 1.11. Constipation problems & $1(0-4)$ & $0(0-4)$ & \multicolumn{2}{|l|}{0.10} \\
\hline 1.12. Lightheadeness on standing & $1(0-3)$ & $0(0-3)$ & \multicolumn{2}{|l|}{0.28} \\
\hline 1.13. Fatigue & $2(0-4)$ & $1(0-4)$ & $0.03^{\mathrm{a}}$ & \\
\hline MDS-UPDRS part $3(/ 132)$ & $30.2( \pm 14.9)$ & $28.1( \pm 12.0)$ & 0.54 & \\
\hline Hoehn \& Yahr stage $(0-5)^{s}$ & $2(1-5)$ & $2(0-4)$ & $0.003^{\mathrm{a}}$ & \\
\hline PAS total $(/ 48)$ & $14.79( \pm 4.69)$ & $3.69( \pm 2.87)$ & $<0.0001^{\mathrm{a}}$ & \\
\hline Part A $(/ 20)$ & $9.47( \pm 4.32)$ & $2.85( \pm 2.87)$ & $<0.0001^{\mathrm{a}}$ & \\
\hline Part B (/16) & $2.38( \pm 2.26)$ & $0.42( \pm 0.85)$ & $<0.0001^{\mathrm{a}}$ & \\
\hline Part C (/12) & $2.94( \pm 2.32)$ & $0.43( \pm 0.85)$ & $<0.0001^{\mathrm{a}}$ & \\
\hline HAMD total $(/ 54)$ & $8.7( \pm 5.2)$ & $4.5( \pm 3.6)$ & $<0.0001^{\mathrm{a}}$ & \\
\hline HARS total $(/ 56)$ & $11.4( \pm 5.8)$ & $5.3( \pm 4.2)$ & $<0.0001^{\mathrm{a}}$ & \\
\hline Cognitive variables & $A+\operatorname{group}(n=34)$ & A- group $(n=84)$ & Uncorrected p-value & FDR-corrected p-value \\
\hline Overall efficiency & & & & \\
\hline MDRS score (/144) & $136( \pm 5.90)$ & $138( \pm 6.62)$ & 0.024 & 0.096 \\
\hline Attention and working memory & & & & \\
\hline$\overline{\text { WAIS-R forward digit (/14) }}$ & $7.12( \pm 2.14)$ & $7.90( \pm 2.19)$ & 0.06 & 0.120 \\
\hline WAIS-R backward digit (/14) & $4.88( \pm 1.87)$ & $6.04( \pm 1.67)$ & 0.001 & $0.008^{\mathrm{a}}$ \\
\hline SDMT: number in $90 \mathrm{~s}$ & $35.35( \pm 10.57)$ & $44.71( \pm 11.91)$ & 0.00009 & $0.001^{\mathrm{a}}$ \\
\hline Executive functions & & & & \\
\hline$\overline{\text { TMT (time } B / \text { time A) }}$ & $2.75( \pm 0.91)$ & $2.47( \pm 0.71)$ & 0.09 & 0.131 \\
\hline Stroop: interference index & $2.04( \pm 0.70)$ & $1.75( \pm 0.47)$ & 0.024 & 0.077 \\
\hline Phonemic fluency: words in $60 \mathrm{~s}$ & $11.97( \pm 4.19)$ & $13.75( \pm 4.84)$ & 0.06 & 0.107 \\
\hline Alternating fluency: words in $60 \mathrm{~s}$ & $10.74( \pm 3.89)$ & $12.04( \pm 4.85)$ & 0.12 & 0.137 \\
\hline Episodic memory & & & & \\
\hline$\overline{\text { HVLT Learn } 1 \text { (/12) }}$ & $5.79( \pm 1.81)$ & $6.64( \pm 1.92)$ & 0.03 & 0.080 \\
\hline HVLT Learn total (/36) & $24.31( \pm 4.30)$ & $26.27( \pm 4.43)$ & 0.04 & 0.091 \\
\hline HVLT delayed recall (/12) & $8.62( \pm 1.92)$ & $9.04( \pm 2.69)$ & 0.09 & 0.120 \\
\hline HVLT recognition hits (/12) & $11.32( \pm 0.88)$ & $11.24( \pm 1.26)$ & 0.78 & 0.780 \\
\hline $\begin{array}{l}\text { HVLT number of intrusions } \\
\text { Language }\end{array}$ & $1.75( \pm 2.05)$ & $1.50( \pm 2.13)$ & 0.37 & 0.395 \\
\hline Boston Naming Test (/15) & $12.26( \pm 2.31)$ & $13.00( \pm 1.96)$ & 0.09 & 0.111 \\
\hline Semantic fluency: animals in 60 s & $16.94( \pm 4.60)$ & $20.71( \pm 5.91)$ & 0.001 & $0.005^{\mathrm{a}}$ \\
\hline Visuospatial functions & $10.71( \pm 3.26)$ & $11.95( \pm 2.55)$ & 0.08 & 0.128 \\
\hline Benton Judgment of Line Orientatic & & & & \\
\hline
\end{tabular}

${ }^{\mathrm{a}}=$ FDR-corrected p-value $<0.05, \S=$ described as median and range; $\mathrm{CI}=$ confidence interval; HAMD $=$ Hamilton Depression Rating Scale; HARS $=$ Hamilton

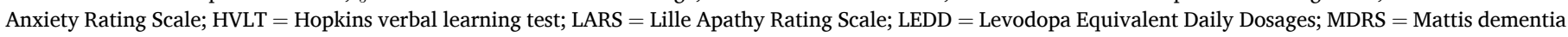

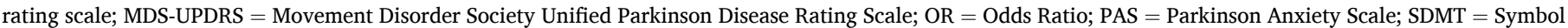
digit modalities test; TMT $=$ Trail Making Test; WAIS-R = Wechsler for adults intelligence scale revised.

\subsection{Functional analyses}

\subsubsection{Functional connectivity of the amygdala}

Of the 118 patients, 17 were excluded from the FC analyses after quality control $(n=101)$. Their demographic and clinical characteristics (presented in Supplementary Table 2) were similar as the original study population.

There were fewer functional connections with both amygdala in the "A+" than in "A-" group. However, after FDR-correction, there was no longer any significant difference (Supplementary Fig. 2 and Supplementary Table 3).
3.5.2. Resting-state functional networks and the fear circuit

In the "A+" group, the FC was significantly higher between the fear circuit and the salience network (F-score $=2.55$, FDR-corrected $\mathrm{p}$-value $=0.0375$ ), compared to "A-" group.

\subsubsection{Regression analyses}

The PAS score was significantly positively related to the FC between the left amygdala-left parahippocampal cortex $(\mathrm{p}=0.010)$. The PAS-B sub-score was significantly negatively related to the mean cortical thickness of the left fronto-cingulate $(\mathrm{p}=0.003)$, right fronto-cingulate $(\mathrm{p}=0.013)$ and left parietal areas $(\mathrm{p}=0.016)$. The PAS-C sub-score was significantly negatively related to the mean cortical thickness of the left fronto-cingulate cluster $(\mathrm{p}=0.016)$ and positively related to the FC 

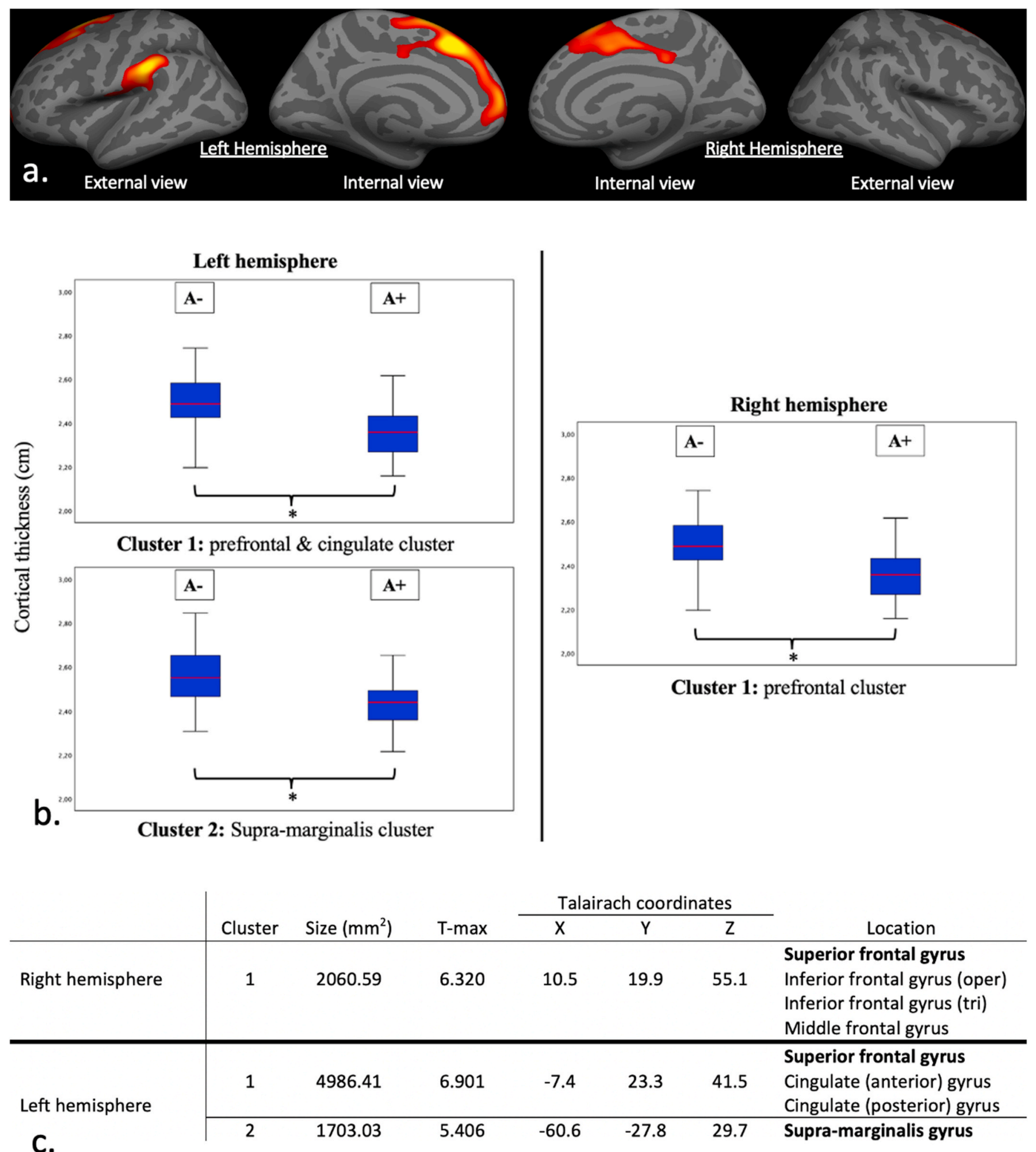

\begin{tabular}{|c|c|c|c|c|c|c|c|}
\hline & \multirow[b]{2}{*}{ Cluster } & \multirow[b]{2}{*}{ Size $\left(\mathrm{mm}^{2}\right)$} & \multirow[b]{2}{*}{$T-\max$} & \multicolumn{3}{|c|}{ Talairach coordinates } & \multirow[b]{2}{*}{ Location } \\
\hline & & & & $x$ & $\mathrm{Y}$ & $Z$ & \\
\hline Right hemisphere & 1 & 2060.59 & 6.320 & 10.5 & 19.9 & 55.1 & $\begin{array}{l}\text { Superior frontal gyrus } \\
\text { Inferior frontal gyrus (oper) } \\
\text { Inferior frontal gyrus (tri) } \\
\text { Middle frontal gyrus }\end{array}$ \\
\hline Left hemisphere & 1 & 4986.41 & 6.901 & -7.4 & 23.3 & 41.5 & $\begin{array}{l}\text { Superior frontal gyrus } \\
\text { Cingulate (anterior) gyrus } \\
\text { Cingulate (posterior) gyrus }\end{array}$ \\
\hline C. & 2 & 1703.03 & 5.406 & -60.6 & -27.8 & 29.7 & Supra-marginalis gyrus \\
\hline
\end{tabular}

Fig. 1. Cortical thickness analysis.

(a) Map of reduced cortical thickness clusters in patients with anxiety compared to patients without anxiety (T-score). (b) Boxplots of cortical thickness comparisons for the four significant clusters of cortical thickness reduction in the Parkinson's disease patients with (A+) and without (A-) anxiety, adjusted by sex. (c) Location and MRI coordinates of cortical thickness atrophy clusters in Parkinson's disease patients with anxiety compared to patients without anxiety.

* = FDR-corrected p-value $<0.05$; Bold $=$ T-max gyrus; oper $=$ opercularis; tri. $=$ triangularis.

between the left amygdala-left parahippocampal cortex $(\mathrm{p}=0.011)$. There were no other significant associations. (Fig. 3 and Supplementary Table 4).

\section{Discussion}

The present study sought to identify anatomical and functional markers of PD-related anxiety. We observed reduced cortical thickness 


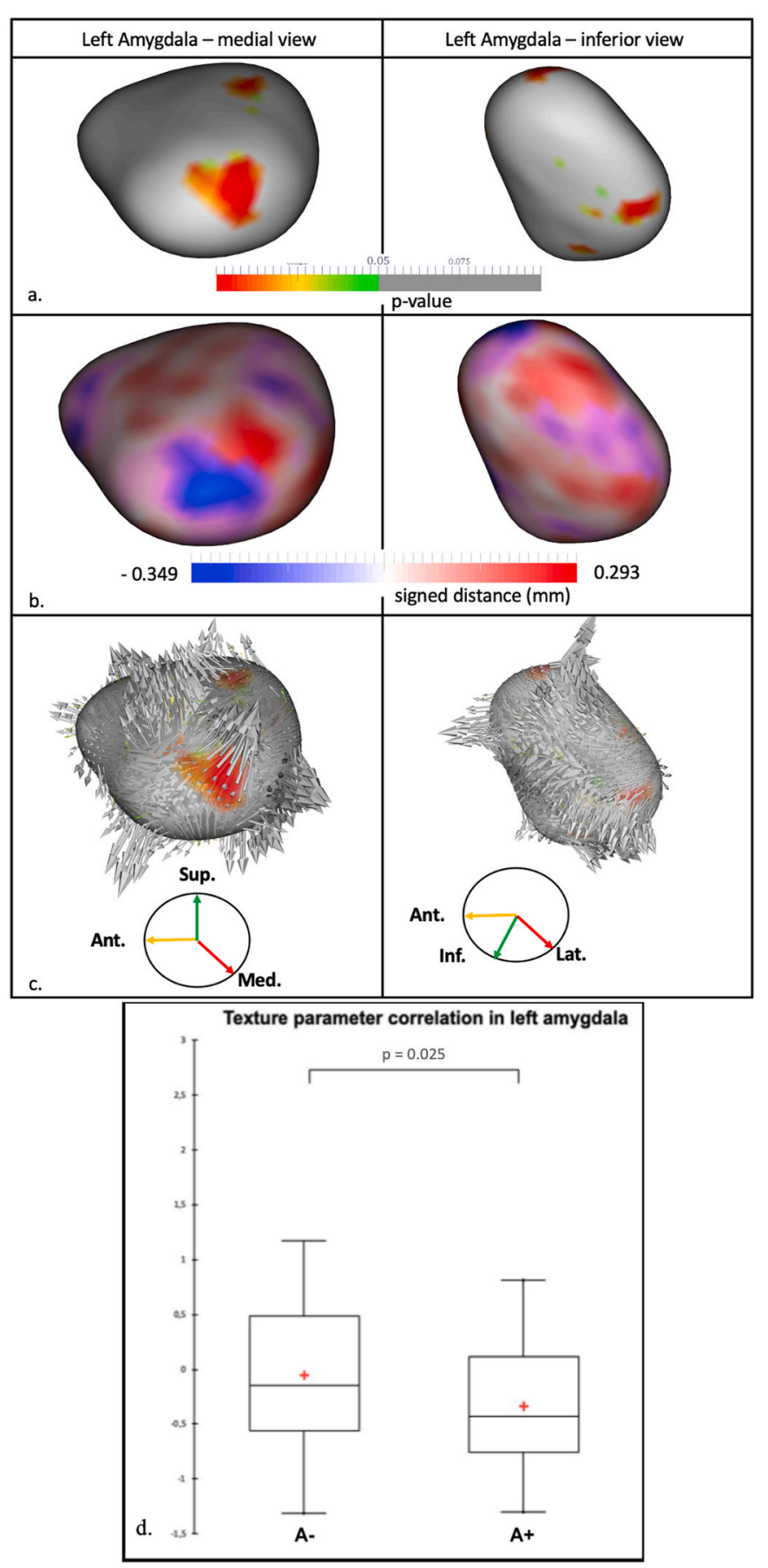

Fig. 2. Anatomical changes of left amygdala in A+ compared to A-patients in PD, adjusted by center, sex and Hoehn-Yarh stage.

Shape analysis: (a)statistical map, (b) signed distance map (mm) and (c) vertex map showing significative shape differences on the medial and inferior sides of the left amygdala.

Texture analysis: (d) distribution of the second-order texture parameter "correlation" in left amygdala between the two groups.

Ant. = Anterior side; Inf. = Inferior side; Lat. = Lateral side; Med. = Medial side; Sup. = Superior side.

of the bilateral fronto-cingulate and left parietal regions, and anatomical changes of the left amygdala in PD patients with anxiety, with several remodeling areas located on the medial and postero-lateral sides of the left amygdala and changes in texture). Moreover, FC between the fear circuit and the salience network was higher in the " $\mathrm{A}+$ " group.

\subsection{PD-related anxiety is associated with changes in the left amygdala}

A negative correlation between the severity of anxiety in PD and the volume of the left amygdala was previously reported [2]. However, we did not find any between group difference in the volume of the amygdala, this volume was not associated with the severity of anxiety symptoms as measured by the PAS. However, shape analyses revealed a remodeling area at the medial and postero-lateral sides of the left amygdala in the "A+" group. Moreover, this structural remodeling of the left amygdala altered the image texture. The lower "correlation" texture parameter in anxious patients may be interpreted as a reduction of the MRI signal consistency. Overall, these results support the role of the amygdala in PD-related anxiety. However, the rather subtle anatomical modifications suggest that the amygdala is not the only structure involved in PD-related anxiety, but more part of a complex system including the "fear circuit" as well as other structures.

\subsection{Anxiety in $P D$ is associated with cortical atrophy in the fear circuit}

We observed clusters of cortical thinning in the bilateral frontocingulate and left parietal cortices. Moreover, the mean cortical thickness of these clusters was negatively associated with the severity of anxiety, especially for episodic anxiety (PAS-B) and avoidance behavior (PAS-C).

As these areas are parts of the fear circuit, their thinning could contribute to disruption of fear processing and thus promote anxiety. In the A+group, the mPFC, ventrolateral PFC (vlPFC) and dorsolateral PFC (dlPFC) had less cortical thickness. In the fear circuit, the vlPFC would be involved in salience detection and action inhibition whereas the dlPFC would be involved in allocation of attentional resources to salient information and cognitive regulation [20-22]. Authors suggested an impaired voluntary emotion regulation by the lateral PFC along with an increased automatic emotion regulation by the MPFC in PD patients with anxiety [4]. These regions are also involved in more general cognitive processes and related to cognitive deficits in PD [23]. Furthermore, smaller cortical thickness of the cingulate cortex could contribute to intrusive negative thoughts that may underlie anxiety symptoms [3]. It could lead to attentional resources disturbance, as found in this study. The parietal cortex has also been involved in PD-related anxiety (precuneus, supramarginalis cortex) [3]. It would be involved in internal awareness and adaptation after environment changes, which possibly explains the difficulty of anxious individuals diverting attention from their negative thoughts [5,24].

\subsection{PD-related anxiety is associated with changes in the FC between networks}

The FC between the "fear circuit" and the salience network was significantly increased in the "A+" group. The salience network is involved in stimuli identification in order to adapt behavior. It is an interface between cognition, emotion and somatic manifestations. It is therefore involved in "bottom-up" attentional processing and could lead to hypervigilance in case of insufficient filtering of these stimuli [25]. These results suggest that any event (e.g. changes in habits, unexpected situations, ...) would be disproportionately perceived in "A+" patients. It would then promote anxious manifestations by letting intrusive thoughts and negative emotions to occur. It could worse anxiety in a vicious circle.

We also observed a trend toward a significant between-group difference of the FC of the amygdala, as well as a positive association between the severity of anxiety and the FC of the left amygdala with the left parahippocampal gyrus, especially for avoidance behavior (PAS-C). Similar associations have been previously reported [5]. Hence, anxiety in PD is associated with a higher activation of the brain fear circuit, led by a higher temporo-amygdala connectivity, which could interfere with other structures. 


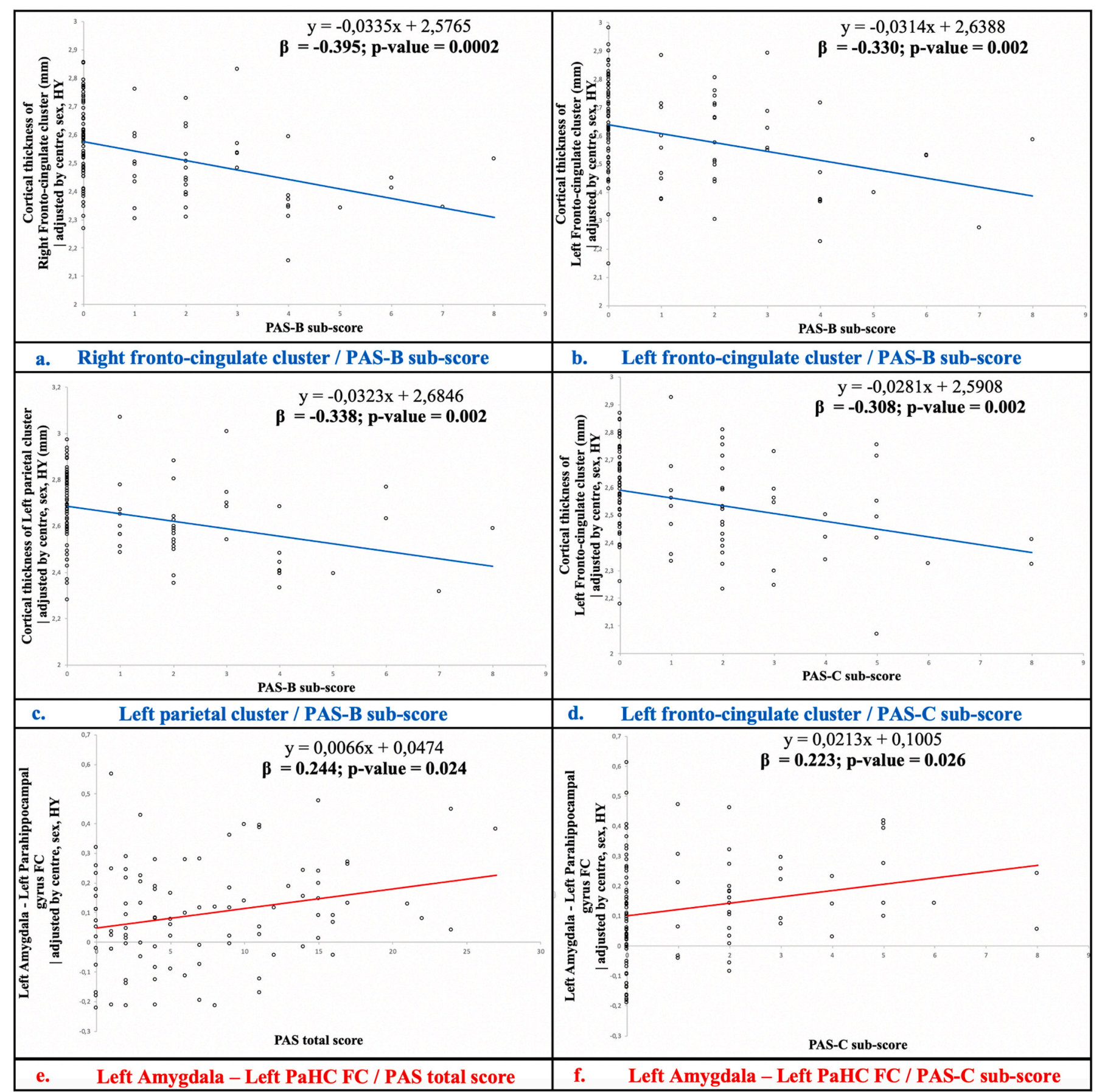

Fig. 3. Regression of the PAS score and sub-scores with the mean cortical thickness of the right fronto-cingulate (a), the left fronto-cingulate (b, d), the left parietal cluster (c) and with the functional connectivity values between the left amygdala and left parahippocampal cortex (e, $f$ ).

Abbreviation: $\mathrm{HY}=$ Hoehn \& Yahr stage; PaHC $=$ parahippocampal cortex; PAS $=$ Parkinson Anxiety Scale.

\subsection{Anxiety is associated with clinical and cognitive features in $P D$}

Female gender, left-sided motor symptoms onset, severity of the disease, the presence of other non-motor symptoms and higher drug use have already been associated with PD-related anxiety [26,27]. Only few studies examined cognitive features of PD patients with anxiety. In this study, "A+" patients had lower scores in attention, working memory and language. This is in line with a previous study showing that, in PD, state anxiety predicts performance in these cognitive domains [28]. Anxiety could thus worsen PD-associated cognitive dysfunctions. We hypothesize that by focusing their attention on anxiety-inducing topics, anxious patients would divert attentional resources, leading to less efficient cognitive control. In return, these cognitive difficulties may increase anxiety. However, the place of cognitive impairment as cause or consequence of anxiety symptoms in PD remains very controversial [29, 30].

\subsection{Limitations}

Firstly, the "A+" patients were considered to have significant anxiety symptoms according to their score at the PAS but did not have a formal diagnosis of any specific anxiety disorder according to diagnostic criteria. However, the PAS has demonstrated high sensitivity and specificity as a diagnostic test for anxiety disorders in PD [11]. Secondly, 
anxiety is a continuous symptom. Interpreting it using a cut-off value could be a potential limitation since a certain proportion of subjects obtained a score close to this threshold (the distribution is shown in Supplementary Fig. 3). Thirdly, despite between-group differences, statistical analyses were not adjusted on medication status and depression. As both groups had similar severity of motor symptoms, it is highly probable that the difference in LEDD was related to anxiety. Introducing it as a covariate would have reduce the effect of anxiety. Regarding depression, only few patients had clinically relevant depressive symptoms and we considered that such correction would have distort reality. Finally, the lack of a healthy control group did not enable us to determine which findings are specific to PD and which are for anxiety in general.

\section{Conclusion}

Structural and functional changes in the human brain fear circuit, including the amygdala, the fronto-cingulate and parietal cortex, play a role in anxiety in PD. These changes could also explain associated cognitive features, such as lower attention. However, alterations within the fear circuit are probably not the only mechanism. Further studies are needed to better explain its link with the physiopathology of the disease.

Funding

This study used data from a study sponsored by the Michael J. Fox Foundation for Parkinson's Research. The sponsor was not involved in the design of this analysis, nor in data interpretation, the writing of this paper or decision to submit this article for publication.

\section{Authors credits}

1) Research project: A. Conception, B. Organization, C. Execution;

2) Statistical Analysis: A. Design, B. Execution, C. Review and Critique;

3) Manuscript: A. Writing of the first draft, B. Review and Critique.

Guillaume Carey (1A/B/C, 2A/B, 3A); Renaud Lopes (1B/C, 2B/C, $3 \mathrm{~B}$ ); Romain Viard (1C, 2B/C, 3B); Nacim Betrouni (1C, 2B/C, 3B); Gregory Kuchcinski (2B/C, 3B); Quentin Devignes (2B/C, 3B); Luc Defebvre (1A, 2C, 3B); Albert FG Leentjens (1A, 2C, 3B); Kathy Dujardin $(1 \mathrm{~A} / \mathrm{B}, 2 \mathrm{~A} / \mathrm{C}, 3 \mathrm{~B})$.

\section{Trial registration}

Clinical Trials Identifier: NCT01792843.

\section{Data availability}

Data supporting the findings of this study are available from the corresponding author, upon reasonable request.

\section{Ethics}

Written informed consent was obtained from all participants after full information of the procedure. The study was approved by the institutional ethics committees (CPP Nord-Ouest IV, 2012-A 01317-36). Additional information on this study group is detailed in the original paper.

\section{Disclosure of competing interest}

No author had conflict of interests related to this study.

G. Carey was employed by Lille University Medical Center and received the EURON mobility grant which was not related to this study.

R. Lopes, R. Viard, G. Kuchcinski and Q. Devignes were employed by Lille University Medical Center and had no other financial disclosure.
N. Betrouni was employed by INSERM (institut national de la santé et de la recherche médicale), received a grant from PROCOPE Campus France and gave consultancies to LLL France.

L. Defebvre was employed by French Government (enseignement supérieur), gave consultancies to "Abbvie" and "Orkyn" and earned honoraria for lectures from "UCB" and "Abbvie".

A.F.G. Leentjens was employed by Maastricht University Medical Center, received a grant from "Michael J Fox Foundation for Parkinson's Research" and earned royalties from "Springer media" and "de Tijdstroom".

K. Dujardin was employed by French Government (enseignement supérieur) and had no other financial disclosure.

\section{Acknowledgment}

The authors thank all participants of the study for their patience and cooperation, Marie Delliaux, Anja Moonen for their help in neuropsychological assessment, Eugenie Mutez for her help in clinical assessment and the "In-vivo Imaging and Functions core facility" team (CI2C http://www.ci2c.fr) for the MRI acquisitions and their help in image processing.

\section{Appendix A. Supplementary data}

Supplementary data to this article can be found online at https://doi. org/10.1016/j.parkreldis.2020.09.020.

\section{References}

[1] M.P.G. Broen, N.E. Narayen, M.L. Kuijf, N.N.W. Dissanayaka, A.F.G. Leentjens, Prevalence of anxiety in Parkinson's disease: a systematic review and metaanalysis, Mov. Disord. 31 (2016) 1125-1133, https://doi.org/10.1002/mds. 26643.

[2] C. Vriend, P.S. Boedhoe, S. Rutten, H.W. Berendse, Y.D. van der Werf, O.A. van den Heuvel, A smaller amygdala is associated with anxiety in Parkinson's disease: a combined FreeSurfer-VBM study, J Neurology Neurosurg Psychiatry 87 (2016) 493-500, https://doi.org/10.1136/jnnp-2015-310383.

[3] N. Wee, M.-C. Wen, N. Kandiah, R.J. Chander, A. Ng, W.L. Au, L.C.S. Tan, Neural correlates of anxiety symptoms in mild Parkinson's disease: a prospective longitudinal voxel-based morphometry study, J. Neurol. Sci. 371 (2016) 131-136, https://doi.org/10.1016/j.jns.2016.10.021.

[4] R. Dan, F. Růžička, O. Bezdicek, E. Rủžička, J. Roth, J. Vymazal, G. Goelman, R. Jech, Separate neural representations of depression, anxiety and apathy in Parkinson's disease, Sci Rep-Uk 7 (2017) 12164, https://doi.org/10.1038/s41598017-12457-6.

[5] H. Zhang, Y. Qiu, Y. Luo, P. Xu, Z. Li, W. Zhu, Q. Wu, W. Tao, Q. Guan, F. Chen, The relationship of anxious and depressive symptoms in Parkinson's disease with voxelbased neuroanatomical and functional connectivity measures, J. Affect. Disord. 245 (2019) 580-588, https://doi.org/10.1016/j.jad.2018.10.364.

[6] P.C. Leal, T.C. Goes, L.C.F. da Silva, F. Teixeira-Silva, Trait vs. state anxiety in different threatening situations, Trends Psychiatry Psychotherapy 39 (2017) 147-157, https://doi.org/10.1590/2237-6089-2016-0044.

[7] P. Tovote, J.P. Fadok, A. Lüthi, Neuronal circuits for fear and anxiety, Nat. Rev. Neurosci. 16 (2015) 317-331, https://doi.org/10.1038/nrn3945.

[8] A.K. Roy, Z. Shehzad, D.S. Margulies, A.M.C. Kelly, L.Q. Uddin, K. Gotimer, B. B. Biswal, F.X. Castellanos, M.P. Milham, Functional connectivity of the human amygdala using resting state fMRI, Neuroimage 45 (2009) 614-626, https://doi. org/10.1016/j.neuroimage.2008.11.030.

[9] L.M. Shin, I. Liberzon, The neurocircuitry of fear, stress, and anxiety disorders, Focus 9 (2011) 311-334, https://doi.org/10.1176/foc.9.3.foc311.

[10] M. Emre, D. Aarsland, R. Brown, D.J. Burn, C. Duyckaerts, Y. Mizuno, G.A. Broe, J. Cummings, D.W. Dickson, S. Gauthier, J. Goldman, C. Goetz, A. Korczyn, A. Lees, R. Levy, I. Litvan, I. McKeith, W. Olanow, W. Poewe, N. Quinn, C. Sampaio, E. Tolosa, B. Dubois, Clinical diagnostic criteria for dementia associated with Parkinson's disease, Mov. Disord. 22 (2007) 1689-1707, https://doi.org/10.1002/ mds. 21507.

[11] A.F.G. Leentjens, K. Dujardin, G.M. Pontone, S.E. Starkstein, D. Weintraub, P. Martinez-Martin, The Parkinson Anxiety Scale (PAS): development and validation of a new anxiety scale, Mov. Disord. 29 (2014) 1035-1043, https://doi. org/10.1002/mds.25919.

[12] K. Dujardin, A.J.H. Moonen, H. Behal, L. Defebvre, A. Duhamel, A.A. Duits, L. Plomhause, C. Tard, A.F.G. Leentjens, Cognitive disorders in Parkinson's disease: confirmation of a spectrum of severity, Park. Relat. Disord. 21 (2015) 1299-1305, https://doi.org/10.1016/j.parkreldis.2015.08.032.

[13] O. Esteban, C.J. Markiewicz, R.W. Blair, C.A. Moodie, A.I. Isik, A. Erramuzpe, J. D. Kent, M. Goncalves, E. DuPre, M. Snyder, H. Oya, S.S. Ghosh, J. Wright, J. Durnez, R.A. Poldrack, K.J. Gorgolewski, fMRIPrep: a robust preprocessing 
pipeline for functional MRI, Nat. Methods 16 (2019) 111-116, https://doi.org/ 10.1038/s41592-018-0235-4.

[14] A. Siozopoulos, V. Thomaidis, P. Prassopoulos, A. Fiska, In vivo estimation of normal amygdala volume from structural MRI scans with anatomical-based segmentation, Surg. Radiol. Anat. 40 (2018) 145-157, https://doi.org/10.1007/ s00276-017-1915-y.

[15] M. Styner, I. Oguz, S. Xu, C. Brechbühler, D. Pantazis, J.J. Levitt, M.E. Shenton, G. Gerig, Framework for the statistical shape analysis of brain structures using SPHARM-PDM, Insight J (2006) 242-250.

[16] R.M. Haralick, K. Shanmugam, I. Dinstein, Textural features for image classification, Ieee Transactions Syst Man Cybern (1973) 610-621, https://doi.org/ 10.1109/tsmc.1973.4309314. SMC-3.

[17] N. Betrouni, R. Lopes, L. Defebvre, A.F.G. Leentjens, K. Dujardin, Texture features of magnetic resonance images: a marker of slight cognitive deficits in Parkinson's disease, Mov Disord Official J Mov Disord Soc 35 (2020) 486-494, https://doi.org/ 10.1002/mds. 27931.

[18] S. Whitfield-Gabrieli, A. Nieto-Castanon, Conn: a functional connectivity toolbox for correlated and anticorrelated brain networks, Brain Connect. 2 (2012) 125-141, https://doi.org/10.1089/brain.2012.0073.

[19] R.S. Desikan, F. Ségonne, B. Fischl, B.T. Quinn, B.C. Dickerson, D. Blacker, R. L. Buckner, A.M. Dale, R.P. Maguire, B.T. Hyman, M.S. Albert, R.J. Killiany, An automated labeling system for subdividing the human cerebral cortex on MRI scans into gyral based regions of interest, Neuroimage 31 (2006) 968-980, https://doi. org/10.1016/j.neuroimage.2006.01.021.

[20] D.T. Stuss, M.P. Alexander, Executive functions and the frontal lobes: a conceptual view, Psychol. Res. 63 (2000) 289-298, https://doi.org/10.1007/s004269900007.

[21] D.T. Stuss, Frontal lobes and attention: processes and networks, fractionation and integration, J. Int. Neuropsychol. Soc. 12 (2006) 261-271, https://doi.org/ $10.1017 /$ s1355617706060358.
[22] D.T. Stuss, Functions of the frontal lobes: relation to executive functions, J. Int. Neuropsychol. Soc. 17 (2011) 759-765, https://doi.org/10.1017/ s1355617711000695.

[23] C.S. Oosterwijk, C. Vriend, H.W. Berendse, Y.D. van der Werf, O.A. van den Heuvel, Anxiety in Parkinson's disease is associated with reduced structural covariance of the striatum, J. Affect. Disord. 240 (2018) 113-120, https://doi.org/10.1016/j. jad.2018.07.053.

[24] G. Hartwigsen, H.R. Siebner, Joint contribution of left dorsal premotor cortex and supramarginal gyrus to rapid action reprogramming, Brain Stimul 8 (2015) 945-952, https://doi.org/10.1016/j.brs.2015.04.011.

[25] V. Menon, Brain Mapping : salience network, Off. Syst. 2 (2015) 597-611, https:// doi.org/10.1016/b978-0-12-397025-1.00052-x.

[26] A.F.G. Leentjens, K. Dujardin, L. Marsh, P. Martinez-Martin, I.H. Richard, S. E. Starkstein, Symptomatology and markers of anxiety disorders in Parkinson's disease: a cross-sectional study, Mov. Disord. 26 (2011) 484-492, https://doi.org/ 10.1002/mds.23528.

[27] N.N.N.W. Dissanayaka, E. White, J.D. O’Sullivan, R. Marsh, N.A. Pachana, G. J. Byrne, The clinical spectrum of anxiety in Parkinson's disease, Mov. Disord. 29 (2014) 967-975, https://doi.org/10.1002/mds.25937.

[28] K.A.E. Martens, C.R.A. Silveira, B.N. Intzandt, Q.J. Almeida, State anxiety predicts cognitive performance in patients with Parkinson's disease, Neuropsychology 32 (2018) 950-957, https://doi.org/10.1037/neu0000478.

[29] N.N.W. Dissanayaka, R.A. Lawson, A.J. Yarnall, G.W. Duncan, D.P. Breen, T. K. Khoo, R.A. Barker, D.J. Burn, I.-P. study group, Anxiety is associated with cognitive impairment in newly-diagnosed Parkinson's disease, Park. Relat. Disord. 36 (2017) 63-68, https://doi.org/10.1016/j.parkreldis.2017.01.001.

[30] A.J. Petkus, J.V. Filoteo, D.M. Schiehser, M.E. Gomez, G. Petzinger, Worse cognitive performance predicts increased anxiety and depressive symptoms in patients with Parkinson's disease: a bidirectional analysis, Neuropsychology 33 (2018) 35-46, https://doi.org/10.1037/neu0000498. 